\title{
Solving variational inequalities using wavelet methods
}

\author{
Markus Hegland ${ }^{1} \quad$ Dale Roberts ${ }^{2}$
}

(Received 31 January 2011; revised 16 November 2011)

\begin{abstract}
We present a multiscale (or hierarchical) approximation of elliptic variational inequalities where there is no need to develop an explicit mesh refinement strategy. That is, we use wavelets to recast elliptic variational inequalities as constrained quadratic optimisation problems in $\ell_{2}$ which we solve with the primal dual-path following method and the projected gradient algorithm.
\end{abstract}

\section{Contents}

1 Introduction

C950

2 Elliptic variational inequalities

C951

http://journal . austms.org.au/ojs/index.php/ANZIAMJ/article/view/3964 gives this article, (c) Austral. Mathematical Soc. 2011. Published November 24, 2011. ISSN 1446-8735. (Print two pages per sheet of paper.) Copies of this article must not be made otherwise available on the internet; instead link directly to this URL for this article. 
3 Uniform grids

C952

4 Towards an adaptive method

C960

References

C964

\section{Introduction}

Variational inequalities (VI) are a way of formulating certain nonlinear problems in a variational framework. Classic examples include the obstacle problem, Signorini's problem in linear elasticity, stochastic games, and pricing American options. This class of nonlinear problems is an interesting intersection between the areas of inequality constrained quadratic optimisation and variational methods for partial differential equations.

Quite a large body of work exists on the construction of multigrid finite element methods for VI [14] and multigrid methods for constrained quadratic optimization whereby the mesh is adaptively modified to handle the nonlinear problem structure given by the constraints. Using multigrid methods it was also observed numerically that almost the same asymptotic convergence rates (as the number of levels goes to infinity) are observed for the constrained multigrid method as for the unconstrained case [15]. This motivates the aim of this article whereby we present some first steps towards an adaptive algorithm for VI in the spirit of the theoretical framework developed by Cohen et al. $[3,4,5]$. In particular, we illustrate our approach with the example of an obstacle problem but note that these methods apply to more general situations.

We use the following notation. The relation $\mathrm{a} \lesssim \mathrm{b}$ means that $\mathrm{a}$ is bounded by some constant times $b$ uniformly in all parameters on which $a$ and $b$ may depend. We write $\mathrm{a} \sim \mathrm{b}$ to mean that $\mathrm{a} \lesssim \mathrm{b}$ and $\mathrm{b} \lesssim \mathrm{a}$ holds. The spaces $L^{p}(U), 1 \leqslant p \leqslant \infty$, are the usual Lebesgue spaces on a domain $\mathrm{U} \subset \mathbb{R}^{\mathrm{d}}$ and $\mathrm{W}^{\mathrm{k}, \mathfrak{p}} \subset \mathrm{L}^{\mathrm{p}}(\mathrm{U})$, for $\mathrm{k} \in \mathbb{N}$ are the Sobolev spaces of functions 
whose weak derivatives up to order $k$ are bounded in $L^{p}(U)$. We abbreviate $H^{k}(u)=W^{k, 2}(u)$ and $H_{0}^{k}(U)=H^{k}(u) \cap\left\{u:\left.u\right|_{\partial u}=0\right\}$. We write 'a.e.' to mean almost everywhere, and write supp $f$ to denote the length of support of the function $f$.

\section{$2 \quad$ Elliptic variational inequalities}

Let $\mathrm{V}$ be a real Hilbert space, whose inner product and norm are denoted by $(\cdot, \cdot)$ and $\|\cdot\|$ respectively. Let $\mathrm{a}: \mathrm{V} \times \mathrm{V} \rightarrow \mathbb{R}$ be a continuous bilinear form and let $\mathrm{K} \subseteq \mathrm{V}$ be closed, nonempty and convex. Let $\mathrm{V}^{\prime}$ be the dual of $\mathrm{V}$ with pairing between $\mathrm{V}^{\prime}$ and $\mathrm{V}$ denoted by $\langle\cdot, \cdot\rangle$. Given the functional $\ell: \mathrm{V} \rightarrow \mathbb{R}$, the problem

$$
\text { find } u \in K \text { such that } a(u, v-u) \geqslant \ell(v-u), \quad \text { for all } v \in K \text {, }
$$

is called an elliptic variational inequality (of the first kind). If for every $v \in \mathrm{V}, \mathrm{a}(v, v) \geqslant \alpha\|v\|^{2}$ with $\alpha>0$ (that is, coerciveness), then Lions and Stampacchia [18, Theorem 2.1] guaranteed that (1) has a unique solution. Our approach to solving (1) is based on solving an equivalent minimisation problem. Let $\mathcal{E}: \mathrm{V} \rightarrow \mathbb{R}$ be given by $\mathcal{E}(v):=\frac{1}{2} \mathrm{a}(v, v)-\ell(v)$, and consider the problem

$$
\text { find } u \in K: \mathcal{E}(u) \leqslant \mathcal{E}(v), \quad \text { for all } v \in K \text {. }
$$

As $\mathrm{K} \subseteq \mathrm{V}$ is closed, nonempty and convex, the functional $\mathcal{E}$ is strictly convex, continuous and coercive on $\mathbf{K}$. Further, if $\boldsymbol{u}$ solves (2), then $\boldsymbol{u}$ is also a solution to (1) [1]. Throughout this article we illustrate our methods with the following simple example.

Example 1. Take the interval $(0,1)$ and let $\mathrm{V}=\mathrm{H}_{0}^{1}(0,1)$. For $\mathfrak{u}, \boldsymbol{v} \in \mathrm{V}$, take

$$
a(u, v)=\int_{0}^{1} u^{\prime}(\xi) v^{\prime}(\xi) d \xi+\mu \int_{0}^{1} u(\xi) v(\xi) d \xi
$$

with $\mu>0$ and $\ell(v)=\langle f, v\rangle$ for $f \in V^{\prime}=H^{-1}(0,1)$. Finally, take $\chi \in$ $\mathrm{H}^{1}(0,1) \cap \mathrm{C}([0,1])$ with $\chi(0) \leqslant 0$ and $\chi(1) \leqslant 0$ and the set $\mathrm{K}:=\{v \in \mathrm{V}: v \geqslant$ 
$\chi$ a.e. on $(0,1)\}$. With these choices, (1) is a weak formulation of the one dimensional obstacle problem

$$
-u^{\prime \prime}+\mu u=f \text { on }\{u>\chi\} \cap(0,1), \quad u \geqslant \chi \text { on }(0,1),
$$

with boundary conditions $\mathfrak{u}(0)=\mathfrak{u}(1)=0$.

\section{Uniform grids}

Construction of a finite dimensional approximation of a variational inequality in terms of finite elements is now well-known [12] and order of convergence estimates for these approximations have been obtained [11]. We quickly recall the construction of such an approximation. Let $h$ be a parameter converging to 0 and $\left\{V_{h}\right\}_{h}$ be a family of finite dimensional subspaces of $V$. Fix $h$ and assume that $V_{h}$ has a basis of piecewise linear functions $\Phi_{h}:=\left\{\phi_{j}: j \in \mathcal{J}_{h}\right\}$ for some index set $\mathcal{J}_{h}$. Thus an arbitrary element of $v_{h} \in V_{h}$ can be represented as $\sum_{j \in \mathcal{J}_{h}} v_{j} \phi_{j}$ with $v_{j} \in \mathbb{R}$. We use the notation $\mathbf{v}_{h}^{\top} \Phi_{h}:=\sum_{j \in \mathcal{J}_{h}} v_{j} \phi_{j}$ for $\mathbf{v}:=\left\{v_{i}: i \in \mathcal{J}_{h}\right\}$. One also constructs a convex subset $K_{h}$ of $V_{h}$ such that $K_{h}$ reduces to a finite number of constraints on the $v_{j}$ and $K_{h}$ is a 'good' approximation of $\mathrm{K}$ [11]. Then problem (1) is approximated by

find $\mathfrak{u}_{h} \in K_{h}$ such that $a\left(u_{h}, v_{h}-u_{h}\right) \geqslant\left(f, v_{h}-u_{h}\right)$, for all $v_{h} \in K_{h}$.

Lions and Stampacchia [18] showed that problem (5) also possesses a unique solution and an equivalent finite dimensional optimisation problem is

$$
\text { find } u_{h} \in K_{h} \text { such that } \varepsilon\left(u_{h}\right) \leqslant \varepsilon\left(v_{h}\right), \quad \text { for all } v_{h} \in K_{h} \text {. }
$$

In Example $1, a(\cdot, \cdot)$ is symmetric and we obtain the constrained quadratic optimisation problem

$$
\text { minimise } \frac{1}{2} \mathbf{x}^{\top} \mathbf{A} \mathbf{x}-\mathbf{f}^{\top} \mathbf{x}, \quad \text { subject to } \mathbf{x} \geqslant \mathbf{c},
$$


where $\mathbf{x}:=\left\{x_{i}\right\}_{i \in \mathcal{J}_{h}}, \mathbf{A}:=\mathbf{a}\left(\Phi_{h}, \Phi_{h}\right), \mathbf{f}:=\left\langle\mathbf{f}, \Phi_{h}\right\rangle$, and $\mathbf{c}$ is a vector that approximates $K_{h}$ by evaluating $\chi$ at the nodal points of $\left\{\phi_{j}\right\}$ so that $K_{h}:=$ $\left\{\mathbf{x}: x_{i} \geqslant c_{i}, i \in \mathcal{J}_{h}\right\}$. A number of algorithms exist for solving constrained quadratic problems of the form (7). Due to the geometry of $K_{h}$, we set $\Pi$ to be the projection operator onto $K_{h}, \Pi \mathbf{x}:=\left\{\mathbf{u}: \mathfrak{u}_{i}:=\max \left(x_{i}, c_{i}\right)\right\}$. One of the simplest methods of solving (6) is the fixed step-size projected gradient method which is based on the iteration

$$
\mathbf{x}^{(\mathrm{k}+1)}=\Pi\left[\mathbf{x}^{(\mathrm{k})}-\mathrm{k}\left(\mathbf{A} \mathbf{x}^{(\mathrm{k})}-\mathbf{f}\right)\right], \quad \mathbf{x}^{(0)} \in \mathrm{K}_{\mathrm{h}},
$$

with $0<\kappa<2 \alpha /\|\mathbf{A}\|^{2}$. Unfortunately, similar to conjugate gradient methods, the projected gradient algorithm takes a large number of iterations to obtain a solution as its convergence rate is very slow on fine meshes unless good preconditioning is applied.

We now perform a multiscale or hierarchical approximation of the minimization (7) based on the multiscale theory for operator equations $[9,16]$ to obtain a wavelet preconditioning for (7). We recall that for $\mathrm{H}=\mathrm{H}^{\mathrm{s}}(\mathrm{U})$ for $\mathrm{s} \in \mathbb{N}_{0}$, we construct a multiresolution that consists of closed subspaces $S_{j}$ of $\mathrm{H}$ called trial spaces such that they are nested and their union is dense in $\mathrm{H}$,

$$
S_{j_{0}} \subset S_{j_{0}+1} \subset \cdots \subset S_{j} \subset S_{j+1} \cdots \subset S, \quad \overline{\left(\bigcup_{j=j_{0}}^{\infty} S_{j}\right)}=H .
$$

The index $j$ is the refinement level and $j_{0} \in \mathbb{N}_{0}$ is the coarsest level. We assume that these multiresolution spaces $S_{j}$ have the form

$$
S_{j}:=\operatorname{span}\left\{\Phi_{j}\right\}, \quad \Phi_{j}:=\left\{\phi_{j, k}: k \in \Delta_{j}\right\},
$$

for some finite index set $\Delta_{j}$. We assume the set $\left\{\Phi_{j}\right\}_{j=j_{0}}^{\infty}$ is uniformly stable, that is, uniformly in $j$

$$
\|\mathbf{u}\|_{\ell_{2}\left(\Delta_{j}\right)} \sim\left\|\mathbf{u}^{\top} \Phi_{j}\right\|_{H}, \quad \mathbf{u}:=\left\{\mathfrak{u}_{k}\right\}_{k \in \Delta_{j}} \in \ell_{2}\left(\Delta_{j}\right),
$$

where we use the notation $\mathbf{u}^{\top} \Phi_{j}=\sum_{k \in \Delta_{j}} u_{k} \phi_{j, k}$ and $\|\mathbf{u}\|_{\ell_{2}\left(\Delta_{j}\right)}:=\sqrt{\sum_{k \in \Delta_{j}} u_{k}^{2}}$. The collection $\Phi_{j}$ is called a single scale basis, as all its elements live on 
the same scale $\mathfrak{j}$, or a generator basis of the multiresolution. We assume that $\phi_{j, k}$ are compactly supported so that $\operatorname{supp} \phi_{j, k} \sim 2^{-j}$ and scaled so that $\left\|\phi_{j, k}\right\|_{H} \sim 1$. Condition (9) and (11) imply that there exists a matrix

$$
\mathbf{M}_{j, 0}=\left[\mathbf{m}_{r, k}^{j}\right]_{r \in \Delta_{j+1}, k \in \Delta_{j}}
$$

of size $\left(\# \Delta_{j+1}\right) \times\left(\# \Delta_{j}\right)$ such that the two scale relation

$$
\phi_{j, k}=\sum_{r \in \Delta_{j+1}} m_{r, k}^{j} \phi_{j+1, r}, \quad k \in \Delta_{j}
$$

holds giving the matrix-vector refinement equation $\Phi_{j}=\mathbf{M}_{j, 0}^{\top} \Phi_{j+1}$. A basis for $\mathrm{H}$ is constructed from functions that span any complement between successive spaces $S_{j}$ and $S_{j+1}$ :

$$
\operatorname{span}\left(\Phi_{j+1}\right)=\operatorname{span}\left(\Phi_{j}\right) \oplus \operatorname{span}\left(\Psi_{j}\right)
$$

where

$$
\Psi_{j}=\left\{\Psi_{j, k}: k \in \nabla_{j}\right\}, \quad \nabla_{j}:=\Delta_{j} \backslash \Delta_{j} .
$$

We call $\left\{\psi_{j, k}\right\}$ the wavelet functions and they satisfy the refinement equation $\Psi_{j}=\mathbf{M}_{j, 1}^{\top} \Phi_{j+1}$ with $\mathbf{M}_{j, 1}$ of size $\left(\# \Delta_{j+1}\right) \times\left(\# \nabla_{j}\right)$. Further, (13) is equivalent to the fact that $\mathbf{M}_{\mathrm{j}}:=\left[\mathbf{M}_{\mathrm{j}, 0}, \mathbf{M}_{\mathrm{j}, 1}\right]$ is invertible as a mapping from $\ell_{2}\left(\Delta_{\mathrm{j}} \cup \nabla_{\mathrm{j}}\right)$ onto $\ell_{2}\left(\Delta_{j+1}\right)$. It follows that $\mathbf{M}_{j}$ performs a change of basis in the space $S_{j+1}$,

$$
\left[\begin{array}{l}
\Phi_{j} \\
\Psi_{j}
\end{array}\right]=\left[\begin{array}{l}
\mathbf{M}_{j, 0}^{\top} \\
\mathbf{M}_{j, 1}^{\top}
\end{array}\right] \Phi_{j+1}=\mathbf{M}_{j}^{\top} \Phi_{j+1}
$$

called a decomposition identity, and applying the inverse of $\mathbf{M}_{\mathbf{j}}$ to both sides we obtain a reconstruction identity

$$
\Phi_{j+1}=\mathbf{M}_{j}^{-\top}\left[\begin{array}{l}
\Phi_{j} \\
\Psi_{j}
\end{array}\right]=\mathbf{M}_{j, 0}^{-\top} \Phi_{j}+\mathbf{M}_{j, 1}^{-\top} \Psi_{j}
$$

Now fixing a finest resolution level J, we obtain

$$
\operatorname{span}\left(\Phi_{J}\right)=\operatorname{span}\left(\Phi_{j_{0}}\right) \oplus \bigoplus_{j=j_{0}}^{J-1} \operatorname{span}\left(\Psi_{j}\right),
$$


and every $v \in \operatorname{span}\left(\Phi_{\mathrm{J}}\right)$ can be written in single scale representation as

$$
v=\left(\mathbf{v}_{\mathrm{J}}\right)^{\mathrm{T}} \Phi_{\mathrm{J}}=\sum_{\mathrm{k} \in \Delta_{\mathrm{j}}} v_{\mathrm{J}, \mathrm{k}} \phi_{\mathrm{J}, \mathrm{k}}
$$

or in multiscale representation as

$$
v=\left(\mathbf{v}_{j_{0}}\right)^{\top} \Phi_{j_{0}}+\sum_{j=j_{0}}^{J-1}\left(\mathbf{u}_{j}\right)^{\top} \Psi_{j} .
$$

We may pass from the single scale to the multiscale representation by an application of the Wavelet transform $\mathbf{T}_{\mathrm{J}}: \ell_{2}\left(\Delta_{\mathrm{J}}\right) \rightarrow \ell_{2}\left(\Delta_{\mathrm{J}}\right)$ given by

$$
\mathbf{T}_{\mathrm{J}} \mathbf{v}_{\mathrm{J}}=\left[\mathbf{v}_{j_{0}}, \mathbf{u}_{j_{0}}, \ldots, \mathbf{u}_{\mathrm{J}-1}\right]^{\top}
$$

and $\mathbf{T}_{\mathrm{J}}=\mathbf{T}_{\mathrm{J}, \mathrm{J}-1} \cdots \mathbf{T}_{\mathrm{J}, j_{0}}$ where

$$
\mathbf{T}_{\mathrm{J}, \mathrm{j}}=\left[\begin{array}{cc}
\mathbf{M}_{\mathrm{j}} & 0 \\
0 & \mathbf{I}^{\left(\# \Delta_{\mathrm{J}}-\# \Delta_{\mathrm{j}+1}\right)}
\end{array}\right] \in \mathbb{R}^{\left(\# \Delta_{\mathrm{J}}\right) \times\left(\# \Delta_{\mathrm{J}}\right)} .
$$

The inverse transform $\mathbf{T}_{\mathbf{J}}^{-1}$ is constructed in a similar way and maps from the multiscale representation to the single scale representation. Applying $\mathbf{T}_{\mathrm{J}}$ or $\mathbf{T}_{\mathrm{J}}^{-1}$ has complexity of order $\mathcal{O}\left(\# \Delta_{\mathrm{J}}\right)=\mathcal{O}\left(\operatorname{dim} S_{\mathrm{J}}\right)$ uniformly in $\mathrm{J}$ and $\mathbf{T}_{\mathrm{J}}$ is called the Fast Wavelet Transform (FWT). Comparing this approach to a multigrid method we see that it has two favourable features: there is no need to develop explicit mesh refinement strategies; and the different scales are introduced through the translations and dilations of a single function which provides a simpler theoretical analysis [10].

We now return to our initial construction of minimization (7) for Example 1 given by the finite dimensional space $V_{h}$ and connect it with the multiscale representation. Suppose $\mathrm{U}=(\mathrm{a}, \mathrm{b}) \subset \mathbb{R}$ and the basis for $\mathrm{V}_{\mathrm{h}}$ is generated by the functions $\phi_{k}(\xi)=\phi\left(2^{\mathrm{J}} \xi-\mathrm{k}\right)$ where $\phi(\xi)=(1+|\xi|)_{+}$for $\xi \in \mathbb{R}$ and some choice of J. The function $\phi$ is sometimes known as the 'hat function', or in the wavelet setting as the (reverse) biorthogonal B-spline scale function of 
order $(2,2)$. Our construction does not depend on the choice $\phi(\xi)=(1-|\xi|)_{+}$ and applies equally well in the case of higher order biorthogonal B-spline wavelets. For simplicity, we transform the problem so that $(a, b)=\operatorname{supp} \phi$, that is, $(a, b)=(-1,1)$. Notice that we can now relate $V_{h}$ to the single scale space $S_{\mathrm{J}}=\operatorname{span}\left(\Phi_{\mathrm{J}}\right)$ by choosing $\mathrm{h} \sim 2^{-\mathrm{J}}$ where $\mathrm{J}$ is our finest resolution level. Therefore, with these choices we view minimization (7) as problem (6) on the finest resolution $\mathrm{J}$ and since $\mathrm{H}=\mathrm{H}_{0}^{1}(0,1)$ and by our choice of basis functions (that is, $\phi(a)=\phi(b)=0$ ), the number of degrees of freedom $\mathrm{N}=2^{\mathrm{J}+1}-1$ and we approximate $\mathrm{K}_{\mathrm{J}}:=\left\{\mathbf{v}_{\mathrm{J}}: v_{\mathrm{J}, \mathrm{k}} \geqslant \mathrm{c}_{\mathrm{J}, \mathrm{k}}, \mathrm{k} \in \Delta_{\mathrm{J}}\right\}$ where $\mathbf{c}_{\mathrm{J}}:=\left\{\mathbf{c}_{\mathrm{J}, \mathrm{k}}:=\chi\left(\mathrm{k} 2^{-\mathrm{J}}\right): \mathrm{k} \in \Delta_{\mathrm{J}}\right\}$. Applying operator $\mathbf{T}_{\mathrm{J}}$ gives a multiscale representation of minimization (7):

$$
\text { minimise } \frac{1}{2} \mathbf{x}^{\top} \mathbf{P} \mathbf{x}+\mathbf{q}^{\top} \mathbf{x} \text { subject to } \mathbf{G} \mathbf{x} \geqslant \mathbf{c},
$$

where $\mathbf{P}:=\mathbf{T}_{\mathrm{J}}^{-\mathrm{T}} \mathbf{A} \mathbf{T}_{\mathrm{J}}^{-1}, \mathbf{q}:=-\mathbf{T}_{\mathrm{J}}^{-\mathrm{T}} \mathbf{f}$, and $\mathbf{G}=\mathbf{T}_{\mathrm{J}}^{-1}$.

We now make two comments. First, (22) has a fundamentally different structure from (7) as the constraint is now $\mathbf{G x} \geqslant \mathbf{c}$, that is, we must transform $\mathbf{x}$ from the multiscale basis to the single scale basis before testing if it is contained in $\mathrm{K}_{\mathrm{J}}$. Second, suppose we constructed the matrix $\mathbf{G}$, then due to the particular band structure of $\mathbf{M}_{\mathrm{J}}$ and $\mathbf{M}_{\mathbf{J}}^{-1}$ we can estimate that $\mathbf{G}$ contains $\mathcal{O}\left(\mathrm{J} \# \Delta_{\mathrm{J}}\right)$ entries. In practice to obtain $\mathbf{G x}$ one ideally does not construct the matrix $\mathbf{G}$ but instead applies the inverse FWT which is applied in $\mathcal{O}\left(\# \Delta_{\mathrm{J}}\right)$ operations. However, while exploring numerical techniques for VI we decided to solve minimization (7) and (22) by a primal-dual path following method based on the Nesterov-Todd scaling using the optimisation package CVXOPT [7] which solves the cone quadratic program (following their notation)

$$
\text { minimise } \frac{1}{2} x^{\top} P x+c^{\top} x \quad \text { subject to } G x+s=h, A x=b, s \succeq 0,
$$

with $\mathrm{P}$ positive definite. The most expensive part of the algorithm involves 
Table 1: Comparison of time (in seconds) between the finite element approximation and multiscale approximation using CVXOPT.

\begin{tabular}{lrrrrrrr}
\hline Size & 32 & 64 & 128 & 256 & 512 & 1024 & 2048 \\
\hline Finite element & 0.27 & 0.38 & 0.45 & 0.59 & 0.96 & 1.74 & 3.29 \\
Multiscale & 0.28 & 0.37 & 0.60 & 1.18 & 2.84 & 8.06 & 32.79 \\
\hline
\end{tabular}

the solution of a set of linear equations of the form

$$
\left[\begin{array}{ccc}
P & A^{\top} & G^{\top} \\
A & 0 & 0 \\
G & 0 & -W^{\top} W
\end{array}\right]\left[\begin{array}{l}
u_{x} \\
u_{y} \\
u_{z}
\end{array}\right]=\left[\begin{array}{l}
b_{x} \\
b_{y} \\
b_{z}
\end{array}\right]
$$

where $W$ is a positive diagonal scaling matrix (see CVXopt documentation). Due to the structure of minimization (7) and (22), (24) reduces to $2 \times 2$ block systems of the form

$$
\left[\begin{array}{cc}
\mathbf{A} & -\mathbf{I} \\
-\mathbf{I} & -\mathbf{W}^{\top} W
\end{array}\right]\left[\begin{array}{l}
\mathrm{u}_{x} \\
\mathbf{u}_{z}
\end{array}\right]=\left[\begin{array}{l}
\mathrm{b}_{x} \\
\mathrm{~b}_{z}
\end{array}\right], \quad\left[\begin{array}{cc}
\mathbf{P} & -\mathbf{G} \\
-\mathbf{G} & -\mathbf{W}^{\top} \mathrm{W}
\end{array}\right]\left[\begin{array}{l}
\mathrm{u}_{x} \\
\mathbf{u}_{z}
\end{array}\right]=\left[\begin{array}{l}
\mathrm{b}_{x} \\
\mathrm{~b}_{z}
\end{array}\right]
$$

Unfortunately, in the multiscale formulation (22), the need to construct the matrix $\mathbf{G}$ results in the difficulty of the problem being shifted into the constraint. To illustrate this point, in Table 1 consider Example 1 using the finite element approach and the multiscale approach and compare the timings obtained with CVXOPT for different problem sizes. This is very different from the unconstrained situation considered by Dahmen and Kunoth [10], in which the multiscale representation $\mathbf{P}$ is an 'optimally preconditioned' version of $\mathbf{A}$ which ensures a more favourable convergence.

We now return to the theory of constructing an adaptive method and recall that there exists a set of functions $\tilde{\Psi}$ called dual wavelets that are biorthogonal or dual to $\Psi$ in the sense that $\langle\Psi, \tilde{\Psi}\rangle=\mathbf{I}[16$, Theorem 3.1] and in the same way, a set of functions $\tilde{\Phi}$ called dual generators satisfying $\langle\Phi, \tilde{\Phi}\rangle=\mathbf{I}$. We 
have the norm equivalence

$$
\|v\|_{\mathrm{H}^{1}(\mathrm{U})}^{2} \sim \sum_{j=j_{0}-1}^{\infty} 2^{2 j}\left\|\left\langle\tilde{\Psi}_{j}, v\right\rangle\right\|_{\ell_{2}\left(\nabla_{j}\right)}^{2},
$$

which implies that every $v \in \mathrm{H}^{1}(\mathrm{U})$ can be expanded uniquely in terms of the $\Psi$ [16, Corollary 3.1]. Its expansion coefficients $\mathbf{v}$ satisfy $\|v\|_{\mathrm{H}^{1}(\mathrm{U})} \sim$ $\|\mathbf{D v}\|_{\ell_{2}}$ where $\mathbf{D}$ is a diagonal matrix with entries $\mathbf{D}_{(j, k),\left(j^{\prime}, k^{\prime}\right)}=2^{j} \delta_{j, j^{\prime}} \delta_{k, k^{\prime}}$ or alternatively, given by the inverse of the diagonal of $\mathbf{P}$. As such, we assume that $\mathbf{P}$ in (22) is replaced by a rescaled version

$$
\overline{\mathbf{P}}:=\mathbf{D}_{\mathrm{J}}^{-1} \mathbf{T}_{\mathrm{J}}^{-\mathrm{T}} \mathrm{a}\left(\Phi_{\mathrm{J}}, \Phi_{\mathrm{J}}\right) \mathbf{T}_{\mathrm{J}}^{-1} \mathbf{D}_{\mathrm{J}}^{-1},
$$

whose condition number is bounded independently of J. In particular, continuing the situation of Example 1, if $\mu=0$, then we obtain $\overline{\mathbf{P}}=\mathbf{I}_{J}$ under this wavelet preconditioning of $\mathbf{A}$.

As proposed by Schölberl [20], to introduce preconditioning into the projected gradient algorithm, the iteration (8) is modified to

$$
\mathbf{x}^{(\mathrm{k}+1)}=\Pi\left[\mathbf{x}^{(\mathrm{k})}-\alpha \mathbf{C}\left(\mathbf{A} \mathbf{x}^{(\mathrm{k})}-\mathbf{f}\right)\right], \quad \mathbf{x}^{(0)} \in \mathrm{K}_{h},
$$

where $\mathbf{C}$ is some self adjoint positive definite matrix approximating $\mathbf{A}^{-1}$. To accomplish favourable convergence, $\mathbf{C}$ must be chosen so that the spectral condition number $\mathrm{k}\left(\mathbf{C}^{1 / 2} \mathbf{A} \mathbf{C}^{1 / 2}\right)$ as small as possible [10]. Further, one would also like to ensure that the matrix-vector operation $\mathbf{C x}$ and the projection operator $\Pi$, with respect to the $\mathbf{C}^{-1}$ energy norm onto $K_{h}$, has complexity of order $\mathcal{O}\left(\operatorname{dim} V_{h}\right)[20]$. That is, $\Pi \mathbf{x}$ is the element in $K_{h}$ that satisfies

$$
\|\Pi \mathbf{x}-\mathbf{x}\|_{\mathbf{C}^{-1}} \leqslant\|\mathbf{z}-\mathbf{x}\|_{\mathbf{C}^{-1}}, \quad \text { for all } \mathbf{z} \in \mathrm{K}_{\mathrm{h}} .
$$

The matrix $\mathbf{C}:=\left(\mathbf{T}_{\mathbf{J}} \mathbf{D}^{-1}\right)\left(\mathbf{T}_{\mathbf{J}} \mathbf{D}^{-1}\right)^{\top}$ is a candidate for such a preconditioner [10, Remark 2.2] and as $\mathbf{T}_{\mathrm{J}}$ is the FWT, the operation $\mathbf{x} \mapsto \mathbf{C x}$ has complexity $\mathcal{O}\left(\operatorname{dim} S_{\mathrm{J}}\right)$ uniformly in $\mathrm{J}$. 
As the exact projection $\Pi$ is too expensive to compute, we follow Schöberl [20] and replace it with an approximate projection $\tilde{\Pi}$ giving the approximate projected gradient method

$$
\mathbf{x}^{(k+1)}=\tilde{\Pi}\left(\tilde{\mathbf{x}}^{(k)}\right), \quad \tilde{\mathbf{x}}^{(\mathrm{k})}=\mathbf{x}^{(\mathrm{k})}-\alpha \mathbf{C}\left(\mathbf{A} \mathbf{x}^{(k)}-\mathbf{f}\right), \quad \mathbf{x}^{(0)} \in \mathrm{K}_{h} .
$$

Theorem 2 (Schöberl [20]). Let $\mathbf{x}^{(\mathrm{k})}$ be generated by (30), $\mathbf{c}_{\mathbf{A}} \leqslant\|\mathbf{A}\| \leqslant \mathbf{C}_{\mathbf{A}}$, and $\alpha \in\left(0,1 / \mathrm{C}_{\mathbf{A}}\right]$. If $\tilde{\Pi}$ satisfies

$$
\left\|\tilde{\Pi}\left(\mathbf{x}^{(k)}\right)-\tilde{\mathbf{x}}^{(\mathrm{k})}\right\|_{\mathbf{C}^{-1}}^{2} \leqslant \rho_{\Pi}\left\|\mathbf{x}^{(\mathrm{k})}-\tilde{\mathbf{x}}^{(k)}\right\|_{\mathbf{C}^{-1}}^{2}+\left(1-\rho_{\Pi}\right)\left\|\Pi\left(\tilde{\mathbf{x}}^{(k)}\right)-\tilde{\mathbf{x}}^{(\mathrm{k})}\right\|_{\mathbf{C}^{-1}}^{2},
$$

with $\rho_{\Pi} \in[0,1)$, then the estimate

$$
\mathcal{E}\left(\mathbf{x}^{(k+1)}\right) \leqslant \rho \mathcal{E}\left(\mathbf{x}^{(k)}\right)+(1-\rho) \mathcal{E}(\mathbf{x})
$$

holds for every $\mathrm{k} \in \mathbb{N}$ with convergence rate $\rho=1-\frac{1}{2} \alpha \mathrm{c}_{\mathbf{A}}\left(1-\rho_{\Pi}\right)$ and the error in the A-energy norm is bounded by

$$
\left\|\mathbf{x}-\mathbf{x}^{(k)}\right\|_{\mathbf{A}}^{2} \leqslant 2 \rho^{k-1}\left(\mathcal{E}\left(\mathbf{x}^{(1)}\right)-\mathcal{E}(\mathbf{x})\right) .
$$

Taking $\rho_{\Pi}=0$ in Theorem 2 gives the estimate for the case of the exact projection. It follows that one obtains the same error estimator as for the unconstrained case.

Corollary 3. If the sequence $\mathbf{x}^{(\mathrm{k})}$ is generated by (30), then with $\rho$ given by Theorem 2, then the iteration error is bounded by

$$
\left\|\mathbf{x}-\mathbf{x}^{(k+1)}\right\|_{\mathbf{A}}^{2} \leqslant \frac{\rho}{1-\rho}\left(\mathbf{x}^{(k+1)}-\mathbf{x}^{(k)}\right)^{\top}\left(2 \mathbf{f}-\mathbf{A} \mathbf{x}^{(k)}-\mathbf{A} \mathbf{x}^{(k+1)}\right) .
$$

We conclude this section with two remarks. First, Theorem 2 is an estimate that is independent of the highest refinement level J. Second, we now need to construct an approximate projection operator $\tilde{\Pi}$ that satisfies the assumptions of Theorem 2. 


\section{Towards an adaptive method}

The first step in constructing an adaptive wavelet method for the solution of (1) relies on mapping the problem from $V$ onto the sequence space $\ell_{2}$ endowed with inner product $\mathbf{x}^{\top} \mathbf{x}$ for $\mathbf{x} \in \ell_{2}$ and norm $\|\mathbf{x}\|:=\sqrt{\mathbf{x}^{\top} \mathbf{x}}$. Our approach is structured on the adaptive wavelet schemes recently development by Cohen et al. $[3,4]$ and we refer you to those articles for the formal construction in the section. However, heuristically we simply take $\mathrm{J} \rightarrow \infty$ in the framework of Section 3. As such, we obtain a wavelet basis $\Psi:=\left\{\psi_{\lambda}: \lambda \in \mathcal{J}\right\} \subset V$ where the index $\lambda \in \mathcal{J}$ encodes scale, spatial location, and the type of wavelet $\psi_{\lambda}$. We denote by $|\lambda|$ the scale associated with $\psi_{\lambda}$. Again, we consider only compactly supported wavelets and assume that the index set $\mathcal{J}$ has the structure $\mathcal{J}=\mathcal{J}_{\phi} \cup \mathcal{J}_{\psi}$ where $\mathcal{J}_{\phi}$ is finite and indexes the scaling functions on a fixed coarsest level $j_{0}$. $\mathcal{J}_{\psi}$ indexes the wavelets with $|\lambda|>j_{0}$. From the compactness of the supports, it follows that at each level $|\lambda|=j$ the set $\mathcal{J}_{j}:=\{\lambda \in \mathcal{J}:|\lambda|=j\}$ is finite with $\# \mathcal{J}_{j} \sim 2^{\text {jd }}$ where $d$ is the ambient spatial dimension (for example, $\mathbb{R}^{\mathrm{d}}$ ). Finally, we assume that the wavelet basis forms a Riesz basis for $\mathrm{V}$, that is, the analysis operator

$$
\mathbf{T}: \mathrm{V}^{\prime} \rightarrow \ell_{2} ; \quad v \mapsto\left\{\left\langle v, \psi_{\lambda}\right\rangle\right\}_{\lambda \in \mathcal{J}}=:\left\{v_{\lambda}\right\}_{\lambda \in \mathcal{J}}=: \mathbf{v},
$$

is bounded and invertible. We identify $\ell_{2}$ with its dual. The adjoint operator $\mathbf{T}^{\prime}$, called the synthesis operator, is given for $\mathbf{v}:=\left\{v_{\lambda}\right\}_{\lambda \in \mathcal{J}} \in \ell_{2}$ by

$$
\mathbf{T}^{\prime}: \ell_{2} \rightarrow \mathrm{V} ; \quad \mathbf{v} \mapsto \sum_{\lambda \in \mathcal{J}} \nu_{\lambda} \psi_{\lambda}=: \mathbf{v}^{\top} \Psi
$$

Using this framework, we now introduce the functional $E: \ell_{2} \rightarrow \mathbb{R}$ given by $\mathrm{E}(\mathrm{x}):=\mathcal{E}\left(\mathbf{T}^{\prime} \mathbf{x}\right)$ and one can show $\mathrm{E}(\mathbf{x})=\mathcal{E}\left(\mathbf{T}^{\prime} \mathbf{x}\right)=\frac{1}{2} \mathbf{x}^{\top} \mathbf{P} \mathbf{x}-\mathbf{q}^{\top} \mathbf{x}$ where $\mathbf{P}:=\mathbf{T A T}^{\prime} \in \mathcal{L}\left(\ell_{2}, \ell_{2}\right)$ and $\mathbf{q}:=\mathbf{T f} \in \ell_{2}$. Here $\mathbf{P}$ is considered an infinite dimensional matrix, $\mathbf{q}$ as an infinite dimensional vector, and we obtain an infinite dimensional version of minimization (22). The functional $E$ restricted to $K:=\left\{\mathbf{v}: \mathbf{T}^{\prime} \mathbf{v} \in \mathrm{K}\right\}$ retains all of the properties of $\mathcal{E}$ restricted to $\mathrm{K}:$ strict convexity, continuity and coercivity. We conclude that our problem (2) has 
now been mapped from $V$ to $\ell_{2}$ and again, to determine if $\mathbf{v} \in \mathrm{K}$ one must check if $\mathbf{T}^{\prime} \mathbf{v} \in \mathrm{K}$ so the convex set $K \subset \ell_{2}$ is no longer of 'bound type' (that is, $\{\mathbf{x}: \mathbf{x} \geqslant \mathbf{z}\}$ for some $\mathbf{z} \in \ell_{2}$ ). To follow the framework of Cohen et al. [3, 4], we first need to identify an infinite dimensional algorithm that constructively obtains a solution to (2), then we need to construct a computable version that adaptively retains a best $\mathrm{N}$ term approximation.

Let $\Pi_{K}$ be the projection operator that assigns to a given point in $V$ its closest point in $\mathrm{K}$, that is, if $v \in \mathrm{V}$ then $\Pi_{\mathrm{K}} v \in \mathrm{K}$ satisfies $\left\|v-\Pi_{\mathrm{K}} v\right\| \leqslant\|v-u\|$ for all $\mathfrak{u} \in \mathrm{K}$. The operator $\Pi_{\mathrm{K}}$ is well defined and Lipschitz [2]. Choose $\mathfrak{u}_{0} \in \mathrm{K}$ then let $u_{n} \in V$ be a sequence recursively given by

$$
u_{n+1}:=\Pi_{K}\left[u_{n}-\alpha\left(A u_{n}-b\right)\right], \quad n=1,2, \ldots,
$$

for some $\alpha>0$. This projected gradient process is an infinite dimensional version of the projected gradient method and if $0 \leqslant \alpha<\|A\|^{-1}$ then by Goldstein [13], $\mathfrak{u}_{\mathfrak{n}} \rightarrow \mathfrak{u} \in \mathbf{K}$ where $\boldsymbol{u}$ solves (2). Therefore, we have our infinite dimensional algorithm as required. As performed in Section 3, we now map the sequence $\left\{u_{n}\right\}$ to the sequence $\left\{\mathbf{x}^{(\mathfrak{n})}\right\} \in \ell_{2}$ through the use of the operators $\mathbf{T}$ and $\mathbf{T}^{\prime}$. This results in an infinite dimensional version of minimization (22) and we can also obtain an infinite dimensional version of the 'preconditioned' projected gradient algorithm given by the iteration (28). A computable version of this algorithm now needs the following ingredients: an adaptive matrix-vector operation which is provided by the routine APPLY of Cohen et al. [3], a nonlinear approximation of $\mathbf{q}$ which can be obtained by RHS [3], and an approximation $\tilde{\Pi}$ of the projection operator $\Pi$. As mentioned at the end of Section 3, Theorem 2 is independent of $J$ therefore an equivalent theorem can be obtained in the $\ell_{2}$ case. Therefore, we are left with constructing the approximate projection operator $\tilde{\Pi}$. However, it can be shown that $\Pi$ satisfies the assumptions of the results by Cohen et al. [6] and as such, we obtain a computable $\tilde{\Pi}$.

The method has been implemented and Figure 1 displays some results. In the one dimensional examples the solution either follows the constraint or is 

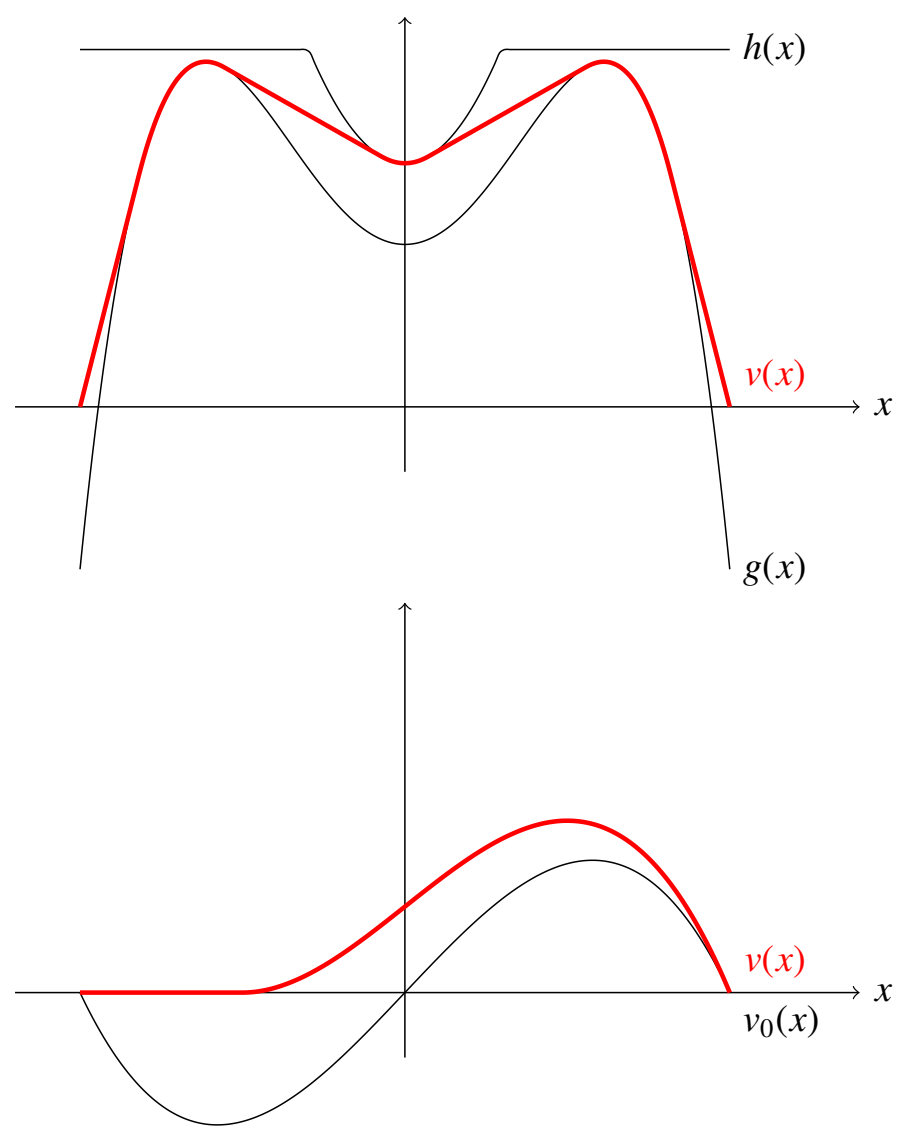

Figure 1: (top) Solution of $-v^{\prime \prime}(x)=0$ with $v(-1)=v(1)=0$ with the constraints $g \leqslant v \leqslant h$ where $g(x)=\left(1-4 x^{2}\right)\left(x^{2}-1 / 2\right)+1$ and $h(x)=$ $3 / 4+4 x^{2}$ when $h(x)<1.1$ and $h(x)=1.1$ otherwise. (bottom) Solution of $-v^{\prime \prime}(x)=x / 10$ with $v(-1)=v(1)=0$ with the positivity constraint $v \geqslant 0$ and $v_{0}$ is the unconstrained solution. 

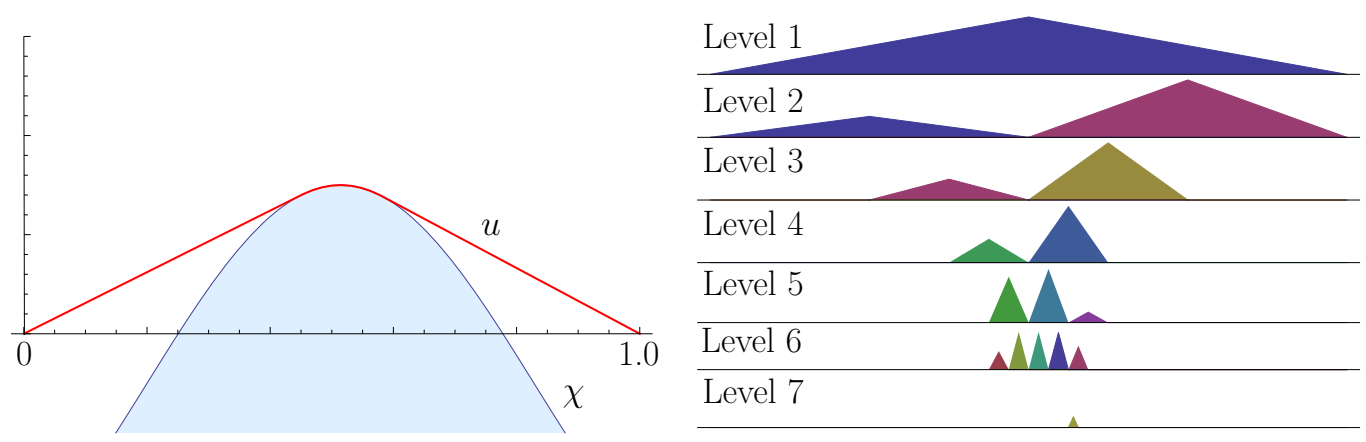

Figure 2: Solution of a one dimensional obstacle problem showing the best 16 term approximation of the solution.

linear (that is, has second derivative zero) and thus solves the constrained problem. These examples demonstrate the feasibility of the approach.

Further investigations are currently being done to assess the performance based on different choices of the approximate projection operator $\tilde{\Pi}$; Lindon Roberts [19] gave a more detailed discussion and additional computational results.

Finally we remark that an adaptive method provides a nonlinear (finite dimensional) approximation of the solution. The efficiency of such approximations is determined by the regularity of the solution in the scale of Besov spaces $B_{q}^{\alpha}\left(L^{p}(U)\right)$ for some bounded, simply connected, Lipschitz domain $\mathrm{U} \subset \mathbb{R}^{\mathrm{d}}$. The higher smoothness of the solution for the Dirichlet problem ensures adaptive methods perform better than linear methods [8]. Therefore, we believe it would be interesting to connect, in a similar way, the regularity theory for the obstacle problem [17, Theorem 3.1] with the rate of convergence of nonlinear approximation. 


\section{References}

[1] Kendall Atkinson and Weimin Han. Theoretical numerical analysis, volume 39 of Texts in Applied Mathematics. Springer, 2 edition, 2005. doi:10.1007/978-0-387-28769-0. C951

[2] Ward Cheney and Allen A. Goldstein. Proximity maps for convex sets. Proc. Amer. Math. Soc., 10:448-450, 1959. C961

[3] Albert Cohen, Wolfgang Dahmen, and Ronald DeVore. Adaptive wavelet methods for elliptic operator equations: convergence rates. Math. Comp., 70(233):27-75 (electronic), 2001. doi:10.1090/S0025-5718-00-01252-7. C950, C960, C961

[4] Albert Cohen, Wolfgang Dahmen, and Ronald DeVore. Adaptive wavelet methods. ii. beyond the elliptic case. Found. Comput. Math., 2(3):203-245, 2002. doi:10.1007/s102080010027. C950, C960, C961

[5] Albert Cohen, Wolfgang Dahmen, and Ronald DeVore. Adaptive wavelet schemes for nonlinear variational problems. SIAM J. Numer. Anal., 41(5):1785-1823 (electronic), 2003. doi:10.1137/S0036142902412269. C950

[6] Albert Cohen, Wolfgang Dahmen, and Ronald DeVore. Sparse evaluation of compositions of functions using multiscale expansions. SIAM J. Math. Anal., 35(2):279-303 (electronic), 2003. doi:10.1137/S0036141002412070. C961

[7] Joachim Dahl and Lieven Vandenberghe. Cvxopt. http://abel.ee.ucla.edu/cvxopt/. C956

[8] Stephan Dahlke and Ronald A. DeVore. Besov regularity for elliptic boundary value problems. Comm. Partial Differential Equations, 22(1-2):1-16, 1997. doi:10.1080/03605309708821252. C963 
[9] Wolfgang Dahmen. Wavelet and multiscale methods for operator equations. Acta numerica, 6:55-228, 1997. C953

[10] Wolfgang Dahmen and Angela Kunoth. Multilevel preconditioning. Numer. Math., 63(3):315-344, 1992. doi:10.1007/BF01385864. C955, C957, C958

[11] Richard S. Falk. Error estimates for the approximation of a class of variational inequalities. Math. Comput., 28:963-971, 1974. C952

[12] Roland Glowinski. Numerical methods for nonlinear variational problems. Scientific Computation. Springer-Verlag, 2008. Reprint of the 1984 original. C952

[13] A. A. Goldstein. Convex programming in hilbert space. Bull. Amer. Math. Soc., 70:709-710, 1964. C961

[14] W. Hackbusch and H.-D. Mittelmann. On multigrid methods for variational inequalities. Numer. Math., 42(1):65-76, 1983. doi:10.1007/BF01400918. C950

[15] Ralf Kornhuber and Rolf Krause. Adaptive multigrid methods for signorini's problem in linear elasticity. Comput. Vis. Sci., 4(1):9-20, 2001. doi:10.1007/s007910100052. C950

[16] Angela Kunoth. Optimized wavelet preconditioning. In Multiscale, nonlinear and adaptive approximation, pages 325-378, 2009. C953, C957, C958

[17] Hans Lewy and Guido Stampacchia. On the regularity of the solution of a variational inequality. Comm. Pure Appl. Math., 22:153-188, 1969. C963

[18] J.-L. Lions and G. Stampacchia. Variational inequalities. Comm. Pure Appl. Math., 20:493-519, 1967. C951, C952

[19] Lindon Roberts. Wavelet methods for variational inequalities, October 2011. Honours Thesis, Australian National University. C963 
[20] J. Schöberl. Solving the signorini problem on the basis of domain decomposition techniques. Computing, 60(4):323-344, 1998. doi:10.1007/BF02684379. C958, C959

\section{Author addresses}

1. Markus Hegland, Mathematical Sciences Institute, Australian National University, Canberra, Australia mailto:markus. hegland@anu . edu . au

2. Dale Roberts, Mathematical Sciences Institute \& School of Finance and Applied Statistics, Australian National University, Canberra, Australia

mailto:dale.roberts@anu.edu.au 